\title{
Nonequilibrium Phase Transition in a Two-Dimensional Driven Open Quantum System
}

\author{
G. Dagvadorj, ${ }^{1}$ J. M. Fellows, ${ }^{1}$ S. Matyjaśkiewicz, ${ }^{2}$ F. M. Marchetti, ${ }^{3}$ I. Carusotto, ${ }^{4}$ and M. H. Szymańska ${ }^{5,}$ \\ ${ }^{1}$ Department of Physics, University of Warwick, Coventry CV4 7AL, United Kingdom \\ ${ }^{2}$ Department of Risk Methodology, Nomura International Plc, 1 Angel Lane, \\ London EC4R 3AB, United Kingdom \\ ${ }^{3}$ Departamento de Física Teórica de la Materia Condensada \\ and Condensed Matter Physics Center (IFIMAC), \\ Universidad Autónoma de Madrid, Madrid 28049, Spain \\ ${ }^{4}$ INO-CNR BEC Center and Dipartimento di Fisica, Università di Trento, I-38123 Povo, Italy \\ ${ }^{5}$ Department of Physics and Astronomy, University College London, \\ Gower Street, London WCIE 6BT, United Kingdom
}

(Received 13 February 2015; revised manuscript received 6 July 2015; published 17 November 2015)

\begin{abstract}
The Berezinskii-Kosterlitz-Thouless mechanism, in which a phase transition is mediated by the proliferation of topological defects, governs the critical behavior of a wide range of equilibrium twodimensional systems with a continuous symmetry, ranging from spin systems to superconducting thin films and two-dimensional Bose fluids, such as liquid helium and ultracold atoms. We show here that this phenomenon is not restricted to thermal equilibrium, rather it survives more generally in a dissipative highly nonequilibrium system driven into a steady state. By considering a quantum fluid of polaritons of an experimentally relevant size, in the so-called optical parametric oscillator regime, we demonstrate that it indeed undergoes a phase transition associated with a vortex binding-unbinding mechanism. Yet, the exponent of the power-law decay of the first-order correlation function in the (algebraically) ordered phase can exceed the equilibrium upper limit: this shows that the ordered phase of driven-dissipative systems can sustain a higher level of collective excitations before the order is destroyed by topological defects. Our work suggests that the macroscopic coherence phenomena, observed recently in interacting twodimensional light-matter systems, result from a nonequilibrium phase transition of the BerezinskiiKosterlitz-Thouless rather than the Bose-Einstein condensation type.
\end{abstract}

DOI: 10.1103/PhysRevX.5.041028

\section{INTRODUCTION}

The Hohenberg-Mermin-Wagner theorem prohibits spontaneous symmetry breaking of continuous symmetries and associated off-diagonal long-range order for systems with short-range interactions at thermal equilibrium in two (or fewer) dimensions [1]. This is because long-range fluctuations due to the soft Goldstone mode are so strong as to be able to "shake apart" any possible long-ranged order. The Berezinskii-Kosterlitz-Thouless (BKT) mechanism (for an overview, see Refs. [2,3]) provides a loophole to the Hohenberg-Mermin-Wagner theorem: Two-dimensional systems can still exhibit a phase transition between a quasi-long-range ordered phase below a critical temperature, where correlations decay

\footnotetext{
* Corresponding author. m.szymanska@ucl.ac.uk

Published by the American Physical Society under the terms of the Creative Commons Attribution 3.0 License. Further distribution of this work must maintain attribution to the author(s) and the published article's title, journal citation, and DOI.
}

Subject Areas: Condensed Matter Physics, Quantum Physics, Superfluidity

algebraically and topological defects are bound together, and a disordered phase above such a temperature, where defects unbind and proliferate, causing exponential decay of correlations. Further, it can be shown [4] that the algebraic decay exponent in the ordered phase cannot exceed the upper bound value of $1 / 4$.

The BKT transition is relevant for a wide class of systems. Perhaps the most celebrated examples are those in the context of 2D superfluids, as in ${ }^{4} \mathrm{He}$ and ultracold atoms: Here, despite the absence of true long-range order, as well as a true condensate fraction, clear evidence of superfluid behavior has been observed in the ordered phase [5]. Particularly interesting, and far from obvious, is the case of harmonically trapped ultracold atomic gases [6]. While, in an ideal gas, trapping modifies the density of states so that to allow Bose-Einstein condensation (BEC) and a true condensate $[7,8]$, weak interactions change the phase transition from normal-to-BEC to normal-tosuperfluid and recover the BKT physics despite the system's harmonic confinement $[9,10]$.

These considerations are applicable to systems in thermal equilibrium, where the BKT transition can be understood in 
terms of the free energy being minimized in either the phase with free vortices or in the one with bound vortex-antivortex pairs. However, in recent years a new class of 2D quantum systems has emerged: strongly driven and highly dissipative interacting many-body light-matter systems such as, for example, polaritons in semiconductor microcavities [11], photons in a dye medium [12], and cold atoms in optical cavities [13] or cavity arrays $[14,15]$. Because of the dissipative nature of the photonic part, a strong drive is necessary to sustain a nonequilibrium steady state. In spite of this, a transition from a normal to a superfluid phase in driven microcavity polaritons has been observed [16,17], and the superfluid properties of the ordered phase have started being explored [18-22]. Being strongly driven, the system does not obey the principle of free energy minimization, and so it is not obvious whether the transition between the normal and superfluid phases, as the density of particles is increased, is of the BKT type, i.e., due to vortexantivortex pairs unbinding.

Current experiments are not yet able to resolve single shot measurements, and so are not sufficiently sensitive to detect randomly moving vortices. Algebraic decay of correlations was reported from averaged data [23,24]; however, the power-law decay displays a larger exponent than the one allowed in equilibrium, which posed questions as to the actual mechanism of the transition. On the theory side, by mapping the complex Ginzburg-Landau equation describing long-wavelength condensate dynamics onto the anisotropic Kardar-Parisi-Zhang equation, Altman et al. [25] concluded that although no algebraic order is possible in a truly infinite system, the Kardar-Parisi-Zhang length scale is certainly much larger than any reasonable system size in the case of microcavity polaritons.

In this work, we consider the case of microcavity polaritons coherently driven into the optical parametric oscillator (OPO) regime [26,27] as the archetype of a nonequilibrium phase transition in a $2 \mathrm{D}$ driven-dissipative system. Another popular pumping scheme is incoherent injection of hot carriers, which relax down to the polariton ground state by exciton formation and interactions with the lattice phonons $[16,17]$. However, the incoherently pumped polariton system is challenging to model due to the complicated and not yet fully understood processes of relaxation. As a result, one is typically forced to use phenomenological models [28], which often suffer from ultraviolet divergences [28]. From this point of view, the parametric oscillation regime is particularly appealing, as an $a b$ initio theoretical description can be developed in terms of a system Hamiltonian [29], and its predictions can be directly compared to experiments.

Analyzing the nonequilibrium steady state with a technique able to account for topological defects and large fluctuations, we show that, despite the presence of a strong drive and dissipation, the transition from the normal to the superfluid phase in this light-matter interacting system is of the BKT type, i.e., governed by binding and dissociation of vortex-antivortex pairs as a function of particle density, and bares a lot of similarities to the equilibrium counterpart. However, as recent experiments suggested [23], we find that larger exponents of the power-law decay of the firstorder correlation function are possible before vortices unbind and destroy the quasi-long-range order leading to exponential decay. This suggests that the external drive, decay, and associated noise favor excitations of collective excitations, the Goldstone phase modes, which lead to faster spatial decay, over unpaired vortices, which would destroy the quasiorder all together. This externally overshaken-but-not-stirred quantum fluid constitutes an interesting new laboratory to explore nonequilibrium phases of matter.

\section{SIMULATING DRIVEN-DISSIPATIVE OPEN SYSTEMS}

We describe the dynamics of polaritons in the OPO regime, by starting from the system Hamiltonian for the coupled exciton and cavity photon field operators $\hat{\psi}_{X, C}(\mathbf{r}, t)$, depending on time $t$ and 2D spatial coordinates $\mathbf{r}=(x, y)(\hbar=1):$

$$
\hat{H}_{S}=\int d \mathbf{r}\left(\begin{array}{ll}
\hat{\psi}_{X}^{\dagger} & \hat{\psi}_{C}^{\dagger}
\end{array}\right)\left(\begin{array}{cc}
\frac{-\nabla^{2}}{2 m_{X}}+\frac{g_{X}}{2}\left|\hat{\psi}_{X}\right|^{2} & \frac{\Omega_{R}}{2} \\
\frac{\Omega_{R}}{2} & \frac{-\nabla^{2}}{2 m_{C}}
\end{array}\right)\left(\begin{array}{l}
\hat{\psi}_{X} \\
\hat{\psi}_{C}
\end{array}\right) .
$$

Here, $m_{X, C}$ are the exciton and photon masses, $g_{X}$ the exciton-exciton interaction strength, and $\Omega_{R}$ the Rabi splitting [11]. In order to introduce the effects of both an external drive (pump) and the incoherent decay, we add to $\hat{H}_{S}$ a system-bath Hamiltonian $\hat{H}_{S B}$

$$
\begin{aligned}
\hat{H}_{S B}= & \int d \mathbf{r}\left[F(\mathbf{r}, t) \hat{\psi}_{C}^{\dagger}(\mathbf{r}, t)+\text { H.c. }\right] \\
& +\sum_{\mathbf{k}} \sum_{l=X, C}\left\{\zeta_{\mathbf{k}}^{l}\left[\hat{\psi}_{l, \mathbf{k}}^{\dagger}(t) \hat{B}_{l, \mathbf{k}}+\text { H.c. }\right]+\omega_{l, \mathbf{k}} \hat{B}_{l, \mathbf{k}}^{\dagger} \hat{B}_{l, \mathbf{k}}\right\},
\end{aligned}
$$

where $\hat{\psi}_{l, \mathbf{k}}(t)$ are obtained Fourier transforming to momentum space $\mathbf{k}=\left(k_{x}, k_{y}\right)$ the corresponding field operators in real space $\hat{\psi}_{l}(\mathbf{r}, t) . \hat{B}_{l, \mathbf{k}}$ and $\hat{B}_{l, \mathbf{k}}^{\dagger}$ are the bath's bosonic annihilation and creation operators with momentum $\mathbf{k}$ and energy $\omega_{l, \mathbf{k}}$, describing the decay processes for both excitons and cavity photons. To compensate the decay, the system is driven by an external homogeneous coherent pump $F(\mathbf{r}, t)=f_{p} e^{i\left(\mathbf{k}_{p} \cdot \mathbf{r}-\omega_{p} t\right)}$, which continuously injects polaritons into a pump state, with momentum $\mathbf{k}_{p}$ and energy $\omega_{p}$.

Within the Markovian bath regime, standard quantum optical methods $[30,31]$ can be used to eliminate the environment and obtain a description of the system dynamics in terms of a master equation. As the full quantum problem is, in practice, intractable, a simple yet 
useful description of the parametric oscillation process properties has been provided by the mean-field approximation, where the quantum fields $\hat{\psi}_{l}(\mathbf{r}, t)$ are replaced by the classical fields $\psi_{l}^{\mathrm{MF}}(\mathbf{r}, t)$, whose dynamics is governed by the following generalized Gross-Pitaevskii equation [11]:

$$
\begin{aligned}
i \partial_{t}\left(\begin{array}{c}
\psi_{X}^{\mathrm{MF}} \\
\psi_{C}^{\mathrm{MF}}
\end{array}\right) & =H_{\mathrm{MF}}\left(\begin{array}{c}
\psi_{X}^{\mathrm{MF}} \\
\psi_{C}^{\mathrm{MF}}
\end{array}\right)+\left(\begin{array}{c}
0 \\
F(\mathbf{r}, t)
\end{array}\right), \\
H_{\mathrm{MF}} & =\left(\begin{array}{cc}
\frac{-\nabla^{2}}{2 m_{X}}+g_{X}\left|\psi_{X}^{\mathrm{MF}}\right|^{2}-i \kappa_{X} & \frac{\Omega_{R}}{2} \\
\frac{\Omega_{R}}{2} & \frac{-\nabla^{2}}{2 m_{C}}-i \kappa_{C}
\end{array}\right),
\end{aligned}
$$

where $\kappa_{X, C}$ are the exciton and photon decay rates. By solving Eq. (1) both analytically and numerically, much work has been carried out on the mean-field dynamics for polaritons in the OPO regime and its properties have been analyzed in detail [32-34]. Here, polaritons resonantly injected into the pump state, with momentum $\mathbf{k}_{p}$ and energy $\omega_{p}$, undergo parametric scattering into the signal $\left(\mathbf{k}_{s}, \omega_{s}\right)$ and idler $\left(\mathbf{k}_{i}, \omega_{i}\right)$ states (see Fig. 1). As explained in detail in Ref. [35], as well as in other works [34], the full steady state OPO photon emission $\psi_{C}^{\mathrm{MF}}(\mathbf{r}, t)$ is filtered in momentum around the values of the signal, pump, and idler momenta $\mathbf{k}_{s, p, i}$ in order to get their corresponding steady state profiles, i.e., $\psi_{s, p, i}^{\mathrm{MF}}(\mathbf{r}, t)=\sum_{\left|\mathbf{k}-\mathbf{k}_{s, p, i}\right|<\tilde{k}_{s, p, i}} \psi_{C, \mathbf{k}}^{\mathrm{MF}}(t) e^{i \mathbf{k} \cdot \mathbf{r}}$. The choice of each state filtering radius $\tilde{k}_{s, p, i}$ is such that the filtered profiles $\psi_{s, p, i}^{\mathrm{MF}}(\mathbf{r}, t)$ are not affected by them; for details, see Ref. [35]. The mean-field onset of OPO is shown in the inset of Fig. 3, where the mean-field densities of both pump and signal [defined as $n_{s, p, i}^{\mathrm{MF}}=\int d \mathbf{r}\left|\psi_{s, p, i}^{\mathrm{MF}}(\mathbf{r}, t)\right|^{2} / V$, with $V$ being the system area] are plotted as a function of the increasing pump power $f_{p}$. Note that the OPO transition also has an upper threshold; i.e., the parametric process is switching off at very high pump powers (see Refs. [32,33] for details). The regime considered in this work is close to the lower threshold for the OPO. At mean-field level, parametric processes lock the sum of the phases of signal and idler fields $\psi_{s, i}^{\mathrm{MF}}$ to that of the external pump, while allowing a global U(1) gauge symmetry for their phase difference to be spontaneously broken in the OPO phase-a feature that implies the appearance of a Goldstone mode [36]. As shown below, fluctuations can lift, close to the OPO threshold, this perfect phase locking.

To move beyond the Gross-Pitaevskii mean-field description [Eq. (1)], one can make use of phase-space techniques-for a general introduction, see Ref. [37], while for recent developments in quantum fluids of atoms and photons, see Refs. [38-40]. Here, the quantum fields $\hat{\psi}_{l}(\mathbf{r}, t)$ are represented as quasiprobability distribution functions in the functional space of $C$-number fields $\hat{\psi}_{l}(\mathbf{r}, t)$. Under suitable conditions, the Fokker-Planck partial differential equation, which governs the time evolution of the quasiprobability distribution, can be mapped on a stochastic

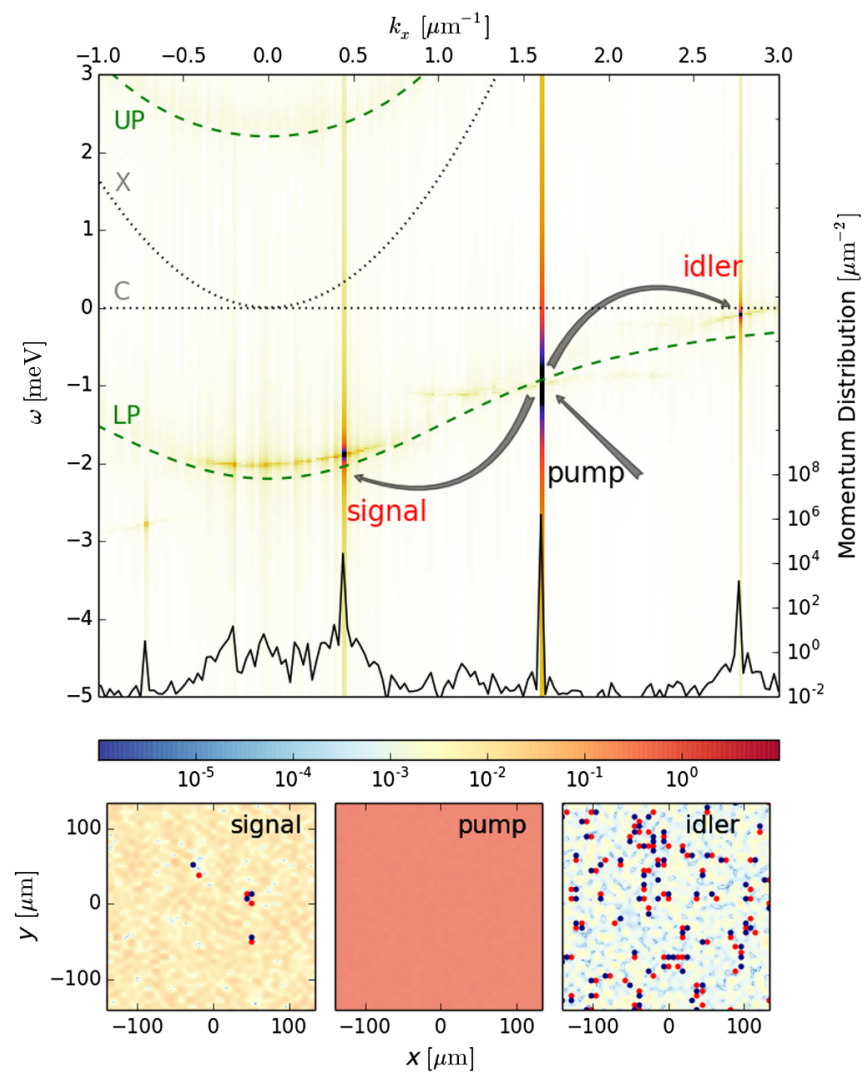

FIG. 1. Polariton system in the OPO regime. Upper panel: 2D map of the photonic OPO spectrum $\left|\psi_{C, k_{x}, k_{y}=0}(\omega)\right|^{2}$ (logarithmic scale) of energy $\omega$ versus the $k_{x}$ momentum component (cut at $k_{y}=0$ ) for a single noise realization and at a pump power $f_{p}=1.02436 f_{p}^{\text {th }}$, where $f_{p}^{\text {th }}$ is the mean-field OPO threshold. The arrows show schematically the parametric process scattering polaritons from the pump state into the signal and idler modes. Dashed (green) lines show the bare upper polariton (UP) and lower polariton (LP) dispersions, while dotted (black) lines are the cavity photon $(C)$ and exciton $(X)$ dispersions. The solid (black) line underneath the spectrum is the $k_{y}=0$ cut of the single-shot time-averaged in the steady state momentum distribution $\int d t\left|\psi_{C, k_{x}, k_{y}=0}(t)\right|^{2}$, clearly showing the macroscopic occupation of the three OPO pump, signal, and idler states. Lower panels: 2D maps of the filtered space profiles $\left|\psi_{s, p, i}(\mathbf{r}, t)\right|^{2}$ at a fixed time $t$ for which a steady state regime is reached-the pump emission intensity is rescaled to 1 . Blue (red) dots indicate the vortex (antivortex) core positions.

partial differential equation, which in turn can be numerically simulated on a finite $N \times N$ grid with spacing $a$ (along both $x$ and $y$ directions) and a total size $L_{x, y}=N a$, comparable to the polariton pump spot size in state-of-theart experiments. For the system under consideration here, the Wigner representation - one of the many possible quasiprobability distributions-is the most suitable to numerical implementation: in the limit $g_{X} /\left(\kappa_{X, C} d V\right) \ll 1$, where $d V=$ $a^{2}$ is the cell area, it in fact appears legitimate [29,41] to truncate the Fokker-Planck equation, retaining the 
second-order derivative term only, thus obtaining the following stochastic differential equation:

$$
i d\left(\begin{array}{l}
\psi_{X} \\
\psi_{C}
\end{array}\right)=\left[H_{\mathrm{MF}}^{\prime}\left(\begin{array}{l}
\psi_{X} \\
\psi_{C}
\end{array}\right)+\left(\begin{array}{c}
0 \\
F
\end{array}\right)\right] d t+i\left(\begin{array}{c}
\sqrt{\kappa_{X}} d W_{X} \\
\sqrt{\kappa_{C}} d W_{C}
\end{array}\right) .
$$

Here, $d W_{l=X, C}$ are complex-valued, zero-mean, independent Wiener noise terms with $\left\langle d W_{l}^{*}(\mathbf{r}, t) d W_{m}\left(\mathbf{r}^{\prime}, t\right)\right\rangle=$ $\delta_{\mathbf{r}, \mathbf{r}^{\prime}} \delta_{l, m}(d t / d V)$, and the operator $H_{\mathrm{MF}}^{\prime}$ coincides with $H_{\mathrm{MF}}$ in Eq. (1) with the replacement $\left|\psi_{X}^{\mathrm{MF}}\right|^{2} \mapsto\left|\psi_{X}\right|^{2}-$ $(1 / d V)$. The same stochastic equation (2) can be alternatively derived applying a Keldysh path integral formalism to the Hamiltonian $\hat{H}_{S}+\hat{H}_{S B}$, integrating out the bath fields, and keeping only the renormalization group relevant terms [42]. Note that, remarkably, some of the difficulties of the truncated Wigner method met in the context of equilibrium systems, such as for cold atoms, are suppressed here by the presence of loss and pump terms, i.e., the existence of a small parameter $g_{X} /\left(\kappa_{l} d V\right)$, which controls the truncation [11]. Note, however, that the bound on this truncation parameter involves the cell area $d V$ of the numerical grid, that is, the UV cutoff of the stochastic truncated Wigner equation. For typical OPO parameters, it is possible to choose $d V$ small enough to capture the physics but at the same time large enough to keep the UV issues under control. Note that this method is particularly suitable for studying the region close to the transition, as it is not limited to small fluctuations, rather it can account for large fluctuations as well as topological defects.

We reconstruct the steady state Wigner distributions $\psi_{l}(\mathbf{r}, t)$ by considering a monochromatic homogeneous continuous-wave pump $F(\mathbf{r}, t)=f_{p} e^{i\left(\mathbf{k}_{p} \cdot \mathbf{r}-\omega_{p} t\right)}$ as before and letting the system evolve to its steady state. In order to rule out any dependence on the chosen initial conditions, we consider four extremely different cases: empty cavity with random noise initial conditions and adiabatic increase of the external pump power strength; mean-field condensate initial conditions; either random or mean-field initial conditions in the presence of an unpumped region at the edges of the numerical box, so as to model a sort of vortexantivortex (V-AV) reservoir. The different initial stage dynamics, and their physical interpretation for each of these four different initial conditions, are carefully described in Ref. [35]; in all four cases we always reach the very same steady state, i.e., all noise-averaged observable quantities, discussed in the following, lead to the same result- this could not be a priori assumed for a nonlinear system.

Below, we first analyze results from single noise realizations (concretely, here, for the case of mean-field initial conditions and no "V-AV reservoir" present), by filtering the photon emission at the signal, pump, and idler momenta as also previously done at the mean-field level. The filtered profiles are again indicated as $\psi_{s, p, i}(\mathbf{r}, t)$; for details on filtering, see Ref. [35]. Second, we consider a large number of independent noise realizations and perform stochastic averages of appropriate field functions in order to determine the expectation values of the corresponding symmetrically ordered quantum operators. In particular, we evaluate the signal first-order correlation function as

$$
g^{(1)}(\mathbf{r})=\frac{\left\langle\psi_{s}^{*}(\mathbf{r}+\mathbf{R}, t) \psi_{s}(\mathbf{R}, t)\right\rangle}{\sqrt{\left\langle\psi_{s}^{*}(\mathbf{R}, t) \psi_{s}(\mathbf{R}, t)\right\rangle\left\langle\psi_{s}^{*}(\mathbf{r}+\mathbf{R}, t) \psi_{s}(\mathbf{r}+\mathbf{R}, t)\right\rangle}},
$$

where the averaging $\langle\cdots\rangle$ is taken over both noise realizations as well as the auxiliary position $\mathbf{R}$, and where $t$ is either a fixed time after a steady state is reached or we take additional time-average in the steady state [35].

\section{VORTICES AND DENSITIES ACROSS THE TRANSITION}

It is particularly revealing to explore the steady state profiles, i.e., $\psi_{s, p, i}(\mathbf{r}, t)$, of the signal, pump, and idler states. Figure 1 shows a cut at $k_{y}=0$ of the OPO spectrum, $\left|\psi_{C, k_{x}, k_{y}=0}(\omega)\right|^{2}$, determined by solving Eq. (2) for $\psi_{X, C}(\mathbf{r}, t)$ to a steady state and evaluating the Fourier transforms in both space and time. The three strongly occupied states, the signal, the pump, and the idler, are clearly visible in the spectrum. Note that the logarithmic scale of this 2D map plot (which we employ to clearly characterize all three OPO states) makes the emission artificially look broad in energy, while in reality this is sharp (as required by a steady state regime), and it is very narrow in momentum. The filtered space profiles $\psi_{s, p, i}(\mathbf{r}, t)$, shown in the bottom panels of Fig. 1, reveal that while the pump state is homogeneous and free from defects, V-AV pairs are present for both signal and idler states. Note that while at the mean-field level the sum of the signal and idler phases is locked to the one of the pump (and thus a V in the signal implies the presence of an $\mathrm{AV}$ at the same position in the idler), the large fluctuations occurring in the vicinity of the OPO threshold make this coherent phase-locking mechanism only weakly enforced, resulting in a different number (and different core locations) of V-AV pairs in the signal and idler states. Because the density of photons in the idler state is much lower than the one at the signal (see, e.g., the photonic momentum distribution plotted as a solid black line inside the upper panel of Fig. 1), while both states experience the same noise strength, the number of $\mathrm{V}-\mathrm{AV}$ pairs in the filtered photonic signal profile is much lower than the number of pairs in the filtered photonic idler profile. Complete phase locking between signal and idler is recovered instead for pump powers well above the OPO threshold, where long-range coherence over the entire pumping region is reestablished.

The proliferation of free vortices below the OPO transition, followed by a sharp decrease in their density 
and their binding into close vortex-antivortex pairs at larger pump powers, is illustrated in Fig. 2. Here, the 2D maps show the phase of the filtered OPO signal $\psi_{s}(\mathbf{r}, t)$ (photonic component) for a single noise realization at increasing values of the pump power $f_{p}$ in a narrow region close to the mean-field OPO threshold $f_{p}^{\text {th }}$; the position of the generated vortices (antivortices) is marked with black (red) dots. While at lower pump powers there is a dense "plasma" of V's and AV's, the number of V's and AV's decreases with increasing pump powers till eventually disappearing altogether (not shown). Note that, as discussed in detail in Ref. [35], due to our momentum filtering procedure we do not detect $\mathrm{V}-\mathrm{AV}$ pairs with distance between $\mathrm{V}$ and $\mathrm{AV}$ smaller than $2.7 \mu \mathrm{m}$, i.e., we focus on free vortices and pairs with relatively large distance between $\mathrm{V}$ and $\mathrm{AV}$, which are relevant for the BKT transition. We also observe a net decrease in the distance between nearest-neighboring vortices with opposite winding number with respect to that between vortices with the same winding number. In order to quantify the vortex binding across the OPO transition, we determine, for each detected vortex, the distance to its nearest vortex, $r_{\mathrm{V}-\mathrm{V}}$ and to its nearest antivortex $r_{\mathrm{V}-\mathrm{AV}}$, and

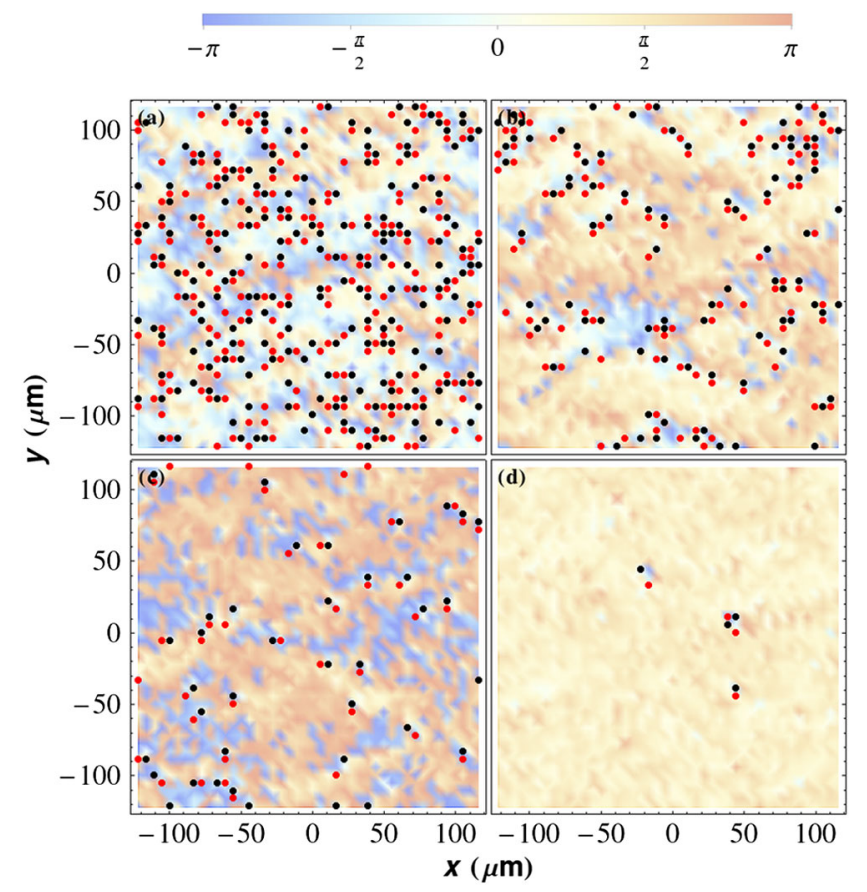

FIG. 2. Binding-unbinding transition and vortex-antivortex proliferation across the OPO threshold. Phase (color map) of the filtered OPO signal $\psi_{s}(\mathbf{r}, t)$ and position of vortices (black dots) and antivortices (red dots) for increasing values of the pump power, in a narrow region close to the mean-field OPO threshold $f_{p}^{\text {th: }}$ (a) $f_{p}=1.00287 f_{p}^{\text {th }}$, (b) $f_{p}=1.01648 f_{p}^{\text {th }}$, (c) $f_{p}=$ $1.01719 f_{p}^{\text {th }}$, and (d) $f_{p}=1.02436 f_{p}^{\text {th }}$. We observe a dramatic decrease of both the number of V's and AV's, as well as the typical distance between $\mathrm{V}$ and $\mathrm{AV}$ within a pair, as a function of the increasing pump power. The filtered profiles are plotted at a late stage of the dynamics, at which a steady state is reached. similarly, for each detected antivortex, we determine $r_{\mathrm{AV}-\mathrm{AV}}$ and $r_{\mathrm{AV}-\mathrm{V}}$. These quantities can then be averaged over many different realizations, as well as over individual vortex positions, to obtain $\left\langle r_{l-m}\right\rangle(l, m=\mathrm{V}, \mathrm{AV})$. We then consider the symmetrized ratio $b=\left(\left\langle r_{\mathrm{V}-\mathrm{AV}}\right\rangle+\left\langle r_{\mathrm{AV}-\mathrm{V}}\right\rangle\right) /$ $\left(\left\langle r_{\mathrm{V}-\mathrm{V}}\right\rangle+\left\langle r_{\mathrm{AV}-\mathrm{AV}}\right\rangle\right)$; this quantity $b \rightarrow 1$ for an unbound vortex plasma, while $b \rightarrow 0$ when vortices form tightly bound pairs. A dramatic drop in $b$ (green squares in Fig. 3) when increasing the pump power across the OPO threshold indicates that vortices and antivortices are indeed binding, as is expected for a BKT transition.

By evaluating other relevant noise-averaged observable quantities, we are able to construct a phase diagram for the OPO transition in Fig. 3 and link it with the properties of the BKT transition. In particular, we calculate the averaged signal photonic density at some time $t$ in the steady state, $n_{s}=\int d \mathbf{r}\left\langle\left|\psi_{s}(\mathbf{r}, t)\right|^{2}\right\rangle / V$, where $V=(N a)^{2}$ is the system area and $\langle\cdots\rangle$ indicates the noise average for the stochastic dynamics (blue dots in Fig. 3). The corresponding meanfield densities for both signal (orange line) and pump (black line) are presented for comparison in the inset of Fig. 3, and additionally for the signal in the main panel (orange

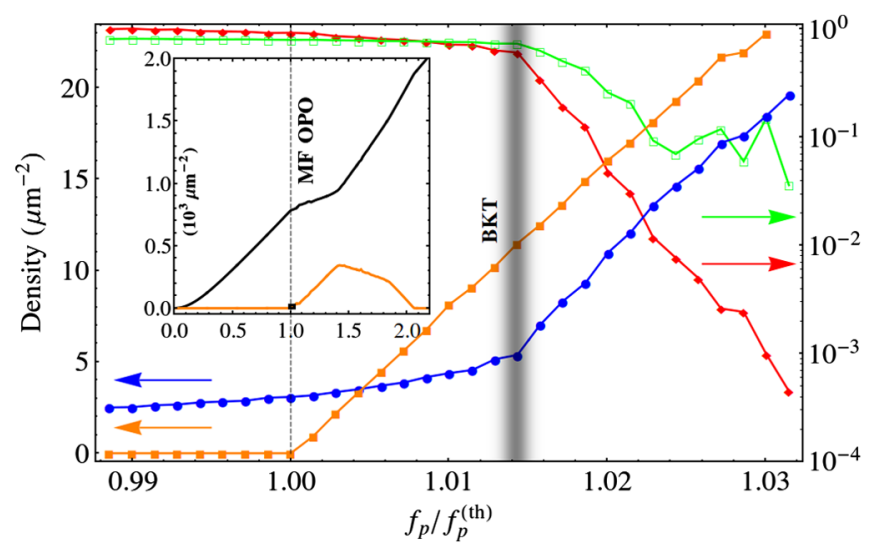

FIG. 3. The phase diagram and the BKT transition. Inset: Mean-field photonic OPO densities for pump $n_{p}^{\mathrm{MF}}$ (black line) and signal $n_{s}^{\mathrm{MF}}$ (orange line) states as a function of increasing pump power $f_{p}$ rescaled by the threshold value $f_{p}^{\text {th }}$ (vertical black dashed line). The black square at $f_{p} \simeq f_{p}^{\text {th }}$ indicates the tiny pump strength interval close to mean-field threshold analyzed in the main panel. Main panel: We plot with (orange) squares the same mean-field signal density $n_{s}^{\mathrm{MF}}$ as in the inset. All other data are noise-averaged properties from stochastic simulations as a function of the pump strength. The noise-averaged signal density $n_{s}$ is plotted with (blue) dots, the average vortex number in the signal rescaled by its average maximum value $N_{\max }=222.8$ with (red) diamonds, and the ratio $b$ of noise-averaged and symmetrized distance between nearest-neighboring vortices of opposite charge and of the same charge with (green) empty squares. The shaded region indicates the pump region for the BKT transition. Note that the left-hand side axis label applies to data marked by orange squares and blue dots, while the right-hand side axis label applies to data marked by red diamonds and green empty squares. 
squares). At mean-field level, both signal and idler (not shown) suddenly switch on at the OPO threshold pump power, $f_{p}=f_{p}^{\text {th }}$, and both states are macroscopically occupied above threshold. The effect of fluctuations is to smoothen the sharp mean-field transition, as clearly shown by comparing $n_{s}$ with $n_{s}^{\mathrm{MF}}$ densities in Fig. 3. This is because, even below the mean-field threshold, incoherent fluctuations weakly populate the signal. Note also that, even though somewhat smoothened, we can still appreciate a kink in the $n_{s}$ density, but at higher values of the pump power compared to the mean-field threshold $f_{p}^{\text {th }}$.

Although defining precisely the position of the critical point in any numerical or experimental study of a finite size system is difficult (see Ref. [35] for more discussion), the behavior of several observables suggests that the critical point for the novel nonequilibrium BKT transition is somewhere around this kink. While this location is further supported by a sudden decrease of the averaged number of detectable vortices in the signal (red diamonds) and of the averaged relative distance $b$ between nearest-neighboring vortices of opposite or same winding number (green squares), the final evidence is offered by the qualitative change in the functional form of the first-order spatial coherence (discussed in the next section) concomitant with the observed kink for $n_{s}$, its long-distance decay changes from an exponential to a power-law form.

These results suggest that the system undergoes a phase transition analogous to the equilibrium BKT transition. Both vortices and antivortices proliferate below some threshold and, above, they bind to eventually disappear altogether. As indicated by the black square in the inset of Fig. 3, the region for such a crossover is indeed narrow in the pump strength, and so a high degree of fine-tuning and control is required in experiments to stay in this regime. Note, however, that as shown in Fig. 3 and further discussed in Ref. [35], this region is no longer so narrow when plotted as a function of the signal polariton density, which changes by an order of magnitude (from 2 to 20 photons per $\mu \mathrm{m}^{2}$ ), and should be experimentally accessible from a standard measurement of the photoluminescence intensity.

\section{FIRST-ORDER SPATIAL CORRELATIONS}

For systems in thermal equilibrium, the BKT transition is associated with the onset of quasi-off-diagonal long-range order, i.e., with the algebraic decay of the first-order correlation function in the ordered phase, where vortices are bound, and exponential decay in the disordered phase, where free vortices do proliferate. In order to investigate whether the same physics applies to the out-of-equilibrium open-dissipative system, we evaluate the signal first-order correlation function $g^{(1)}(\mathbf{r})$ according to the prescription of Eq. (3), and characterize its long-range behavior in Fig. 4. We observe the ordering transition as a crossover in the

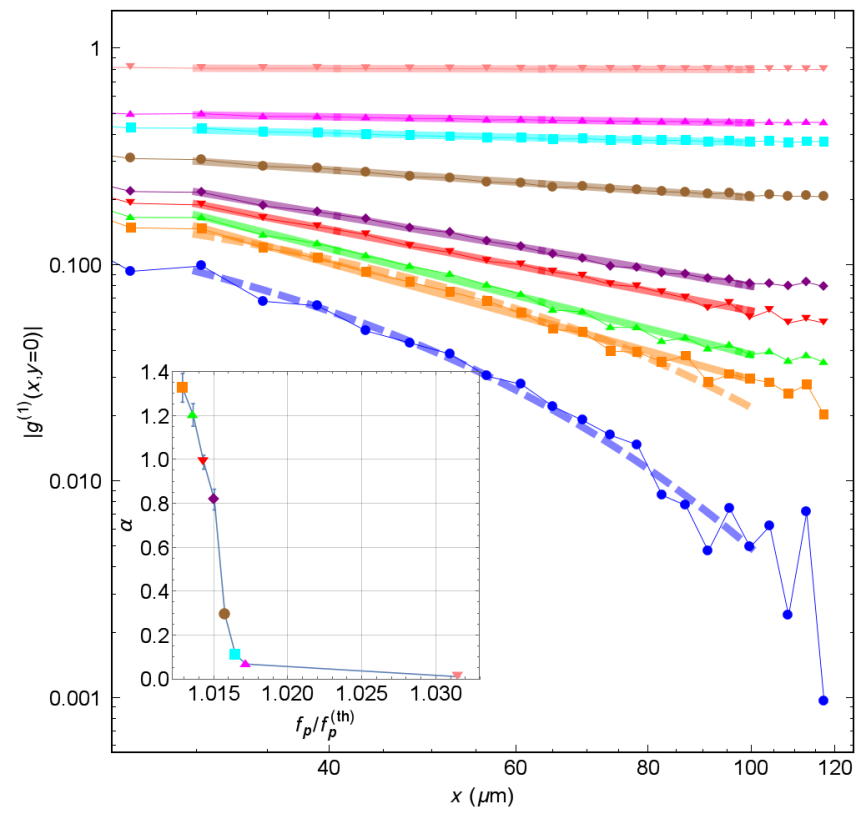

FIG. 4. Algebraic and exponential decay of the first-order correlation function across the BKT transition. Main panel: Long-range spatial dependence of $g^{(1)}(\mathbf{r})$ for different pump powers $f_{p} / f_{p}^{\text {th }}$ close to the mean-field pump threshold (the symbols are the same ones as in the inset and correspond to the same values of $f_{p} / f_{p}^{\text {th }}$ ). Thick solid (thick dashed) lines are power-law (exponential) fitting, from which values of the exponent $\alpha$ are derived. The $f_{p} / f_{p}^{\text {th }}=1.0129$ case (orange squares) is a marginal case where both algebraic and exponential fits apply almost equally well, signaling the BKT transition region. Inset: Power-law algebraic decay exponent $\alpha$ for different pump powers $f_{p} / f_{p}^{\text {th }}$; error bars are standard deviations of the time-average.

long-distance behavior between an exponential decay in the disordered phase, $g^{(1)}(\mathbf{r}) \sim e^{-r / \xi}$, and an algebraic decay in the quasiordered phase, $g^{(1)}(\mathbf{r}) \sim\left(r / r_{0}\right)^{-\alpha}$. We therefore fit the tail of the calculated correlation function to both of these functional forms, and find that at the onset of vortex binding-unbinding and proliferation the signal's spatial correlation function changes its long-range nature from exponential at lower pump powers to algebraic at higher pump powers (see Fig. 4).

However, in contrast to the thermal equilibrium case, we do obtain that the exponent $\alpha$ of the power-law decay (inset of Fig. 4) can exceed the equilibrium upper bound of $1 / 4$ [4], and can reach values as high as $\alpha \simeq 1.2$ for $f_{p} / f_{p}^{\text {th }}=1.0136$, just within the ordered phase. The dependence of $\alpha$ on the system size [35] confirms that its large value is indeed a consequence of the nonequilibrium condition and not an artifact of the finite size of the numerical box. Further, as thoroughly discussed in Ref. [35], it is interesting to note that, close to the transition, we do record a critical slowing-down of the dynamics: Here, the convergence to a steady state is dramatically slowed 
down compared to cases above or below the OPO transition, a feature also common to other phase transitions. At the same time, close to threshold, the convergence of a noiseaveraged number of vortices is much faster than the convergence of the power-law exponent $\alpha$. This indicates that collective excitations take longer time than topological defects to equilibrate to a steady state. Finally, note that for sufficiently strong pump powers, the power-law exponent becomes extremely small, and, thus, quasi-long-range order is difficult to distinguish from the true long-range order over the entire system size.

Our findings explain why recent experimental studies, both in the OPO regime [43] as well as for nonresonant pumping [23], experienced noticeable difficulties in investigating the power-law decay of the first-order correlation function across the transition. We do indeed find that the pump strength interval over which power-law decay can be clearly seen is extremely small, and the system quickly enters a regime where coherence extends over the entire system size, as measured in Ref. [43]. This was also observed in nonresonantly pumped experiments when using a single-mode laser [16]. However, by intentionally adding extra fluctuations by employing a multimode laser pump, as in Ref. [23], power-law decay was finally measured in the correlated regime, with an exponent in the range $\alpha \simeq 0.9-1.2$, in agreement with our results.

We finally note that the exponent $\alpha>1 / 4$ recently observed in the atomic experiment of Ref. [44] can be attributed to a spatial inhomogeneity rather than a nonequilibrium effect: Along these lines, we expect that experimental investigation of our prediction for polariton gases will require using a pump beam with a spatially wide region of homogeneous intensity and a precise control of photonic disorder.

\section{DISCUSSION}

Considering microcavity polaritons in the optical parametric oscillator regime as the prototype of a drivendissipative system, and using methods able to account for large fluctuations and topological defects, we show that a mechanism analogous to the BKT transition, which governs the equilibrium phase transitions in two dimensions, occurs out of equilibrium for a driven-dissipative system of experimentally realistic size. Notwithstanding the novelty and significance of this result, there are a number of novel features that warrant special discussion as they are peculiar to the nonequilibrium phase transition. We show that the exponent of algebraic decay in the quasi-longrange ordered phase exceeds what would be attainable in equilibrium. This recovers a recent observation [23], and strongly suggests that indeed a nonequilibrium BKT may have been seen there. Moreover, our findings imply that before topological defects destroy the order, the quasiordered phase can somehow sustain a higher level of smooth collective excitations in a dissipative-driven nonequilibrium system than in a thermal equilibrium scenario. There are several open questions that stem from our work. Is there a nonequilibrium universal upper bound for the $\alpha$ exponent? What would it depend on and would it smoothly connect to the equilibrium case?.

Although for realistic experimental conditions we find that the region for BKT physics, before the pump power is strong enough to induce perfect spatial coherence over the entire system size, is indeed narrow, we believe our work will encourage further experimental investigations in the direction of studying the nonequilibrium BKT phenomena. Even though the small size of the critical region has thus far hindered its direct experimental study, our calculations indicate that the macroscopic coherence observed in past polariton experiments $[16,17,26,27,43]$ results from a nonequilibrium phase transition of the BKT rather than the BEC kind.

\section{ACKNOWLEDGMENTS}

We thank J. Keeling for stimulating discussions. M. H. S. acknowledges support from EPSRC (Grants No. EP/ I028900/2 and No. EP/K003623/2), F. M. M. from the programs Ministerio de Economia y Competitividad (No. MAT2011-22997) and Comunidad Autonoma de Madrid (No. S-2009/ESP-1503), and I.C. from ERC through the QGBE grant and from the Autonomous Province of Trento, partly through the project "On silicon chip quantum optics for quantum computing and secure communications" ("SiQuro").

\section{APPENDIX: METHODS}

We simulate the dynamics of the stochastic equations (2) with the XMDS2 software framework [46] using a fixedstep (where the fixed-step size ensures stochastic noise consistency) fourth-order Runge-Kutta algorithm, which we have tested against fixed-step ninth-order Runge-Kutta, and a semi-implicit fixed-step algorithm with 3 and 5 iterations. We choose the system parameters to be close to current experiments [20]: The Rabi frequency is chosen as $\Omega_{R}=4.4 \mathrm{meV}$, the mass of the microcavity photons is taken to be $m_{C}=2.3 \times 10^{-5} m_{e}$, where $m_{e}$ is the electron mass, the mass of the excitons is much greater than this so we consider $m_{X}^{-1} \rightarrow 0$, the exciton and photon decay rates as $\kappa_{X}=\kappa_{C}=0.1 \mathrm{meV}$, and the exciton-exciton interaction strength $g_{X}=0.002 \mathrm{meV} \mu \mathrm{m}^{2}$ [47]. The pump momentum $\mathbf{k}_{p}=\left(k_{p}, 0\right)$, with $k_{p}=1.6 \mu \mathrm{m}^{-1}$, is fixed just above the inflection point of the LP dispersion, and its frequency, $\omega_{p}-\omega_{X}(0)=1.0 \mathrm{meV}$, just below the bare LP dispersion. In order to satisfy the condition necessary to derive the truncated Wigner equation $(2), g_{X} /\left(\kappa_{X, C} d V\right) \ll 1$, while maintaining a sufficient spatial resolution and, at the same time, a large enough momentum range so as to resolve the idler state, simulations are performed on a $2 \mathrm{D}$ finite grid of $N \times N=280 \times 280$ points and lattice spacing $a=0.866 \mu \mathrm{m}$. 
Thus, the only system parameter left free to be varied is the pump strength $f_{p}$ : We first solve the mean-field dynamics (1) in order to determine the pump threshold $f_{p}^{\text {th }}$ for the onset of OPO (it is $f_{p}^{\text {th }}=5 \mathrm{meV} / \mu \mathrm{m}$ for the above parameters). We then vary the value of $f_{p}$ around $f_{p}^{\text {th }}$ in the presence of the noise in order to investigate the nature of the OPO transition. We analyze the results from single noise realizations by filtering the full photonic emission for signal pump and idler states, as described in the main text, as well as in Ref. [35]. Further, we average all of our results over many independent realizations, which are taken either from 96 independent stochastic paths or from multiple independent snapshots in time after the steady state is reached: As thoroughly discussed in Ref. [35], 96 stochastic paths is shown to be sufficient to ensure the convergence of noise-averaged observable quantities. For each noise realization, vortices are counted by summing the phase difference (modulo $2 \pi$ ) along each link around every elementary plaquette on the filtered grid. In the absence of a topological defect, this sum is zero, while if the sum is $2 \pi(-2 \pi)$, we determine there to be a vortex (antivortex) at the center of the plaquette. The number of vortices is then averaged over the different stochastic paths or over time in the steady state (see Ref. [35]): We consider the averaged number of vortices to be converged in time when its variation is less than $5 \%$. Finally, the first-order correlation function $g^{(1)}(\mathbf{r})$ is evaluated according to Eq. (3), by averaging over both the noise and the auxiliary position $\mathbf{R}$; as discussed in Ref. [35], this can be computed efficiently in momentum space.

[1] N. Mermin and H. Wagner, Absence of Ferromagnetism or Antiferromagnetism in One- or Two-Dimensional Isotropic Heisenberg Models, Phys. Rev. Lett. 17, 1133 (1966).

[2] P. M. Chaikin and T. C. Lubensky, Principles of Condensed Matter Physics (Cambridge University Press, Cambridge, England, 2000), Vol. 1.

[3] P. Minnhagen, The Two-Dimensional Coulomb Gas, Vortex Unbinding, and Superfluid-Superconducting Films, Rev. Mod. Phys. 59, 1001 (1987).

[4] D. R. Nelson and J. M. Kosterlitz, Universal Jump in the Superfluid Density of Two-Dimensional Superfluids, Phys. Rev. Lett. 39, 1201 (1977).

[5] D. J. Bishop and J. D. Reppy, Study of the Superfluid Transition in Two-Dimensional ${ }^{4}$ He Films, Phys. Rev. Lett. 40, 1727 (1978).

[6] Z. Hadzibabic, P. Krüger, M. Cheneau, B. Battelier, and J. Dalibard, Berezinskii-Kosterlitz-Thouless Crossover in a Trapped Atomic Gas, Nature (London) 441, 1118 (2006).

[7] V. Bagnato and D. Kleppner, Bose-Einstein Condensation in Low-Dimensional Traps, Phys. Rev. A 44, 7439 (1991).

[8] L. Pitaevskii and S. Stringari, Bose-Einstein Condensation, International Series of Monographs on Physics (Clarendon Press, Oxford, 2003).
[9] M. Holzmann, G. Baym, J. Blaizot, and F. Laloe, Superfluid Transition of Homogeneous and Trapped Two-Dimensional Bose Gases, Proc. Natl. Acad. Sci. U.S.A. 104, 1476 (2007).

[10] R. J. Fletcher, M. Robert-de-Saint-Vincent, J. Man, N. Navon, R. P. Smith, K. G. H. Viebahn, and Z. Hadzibabic, Connecting Berezinskii-Kosterlitz-Thouless and BEC Phase Transitions by Tuning Interactions in a Trapped Gas, Phys. Rev. Lett. 114, 255302 (2015).

[11] I. Carusotto and C. Ciuti, Quantum Fluids of Light, Rev. Mod. Phys. 85, 299 (2013).

[12] J. Klaers, J. Schmitt, F. Vewinger, and M. Weitz, BoseEinstein Condensation of Photons in an Optical Microcavity, Nature (London) 468, 545 (2010).

[13] H. Ritsch, P. Domokos, F. Brennecke, and T. Esslinger, Cold Atoms in Cavity-Generated Dynamical Optical Potentials, Rev. Mod. Phys. 85, 553 (2013).

[14] I. Carusotto, D. Gerace, H. E. Tureci, S. De Liberato, C. Ciuti, and A. Imamoğlu, Fermionized Photons in an Array of Driven Dissipative Nonlinear Cavities, Phys. Rev. Lett. 103, 033601 (2009).

[15] M. J. Hartmann, F. G. S. L. Brandao, and M. B. Plenio, Quantum Many-Body Phenomena in Coupled Cavity Arrays, Laser Photonics Rev. 2, 527 (2008).

[16] J. Kasprzak et al., Bose-Einstein Condensation of Exciton Polaritons, Nature (London) 443, 409 (2006).

[17] H. Deng, H. Haug, and Y. Yamamoto, Exciton-Polariton Bose-Einstein Condensation, Rev. Mod. Phys. 82, 1489 (2010).

[18] A. Amo, J. Lefrère, S. Pigeon, C. Adrados, C. Ciuti, I. Carusotto, R. Houdré, E. Giacobino, and A. Bramati, Superfluidity of Polaritons in Semiconductor Microcavities, Nat. Phys. 5, 805 (2009).

[19] A. Amo et al., Collective Fluid Dynamics of a Polariton Condensate in a Semiconductor Microcavity, Nature (London) 457, 291 (2009).

[20] D. Sanvitto et al., Persistent Currents and Quantized Vortices in a Polariton Superfluid, Nat. Phys. 6, 527 (2010).

[21] F. M. Marchetti, M. H. Szymańska, C. Tejedor, and D. M. Whittaker, Spontaneous and Triggered Vortices in Polariton Optical-Parametric-Oscillator Superfluids, Phys. Rev. Lett. 105, 063902 (2010).

[22] G. Tosi et al., Onset and Dynamics of Vortex-Antivortex Pairs in Polariton Optical Parametric Oscillator Superfluids, Phys. Rev. Lett. 107, 036401 (2011).

[23] G. Roumpos et al., Power-Law Decay of the Spatial Correlation Function in Exciton-Polariton Condensates, Proc. Natl. Acad. Sci. U.S.A. 109, 6467 (2012).

[24] G. Roumpos and Y. Yamamoto, Exciton Polaritons in Microcavities (Springer, New York, 2012), pp. 85-146.

[25] E. Altman, L. M. Sieberer, L. Chen, S. Diehl, and J. Toner, Two-Dimensional Superfluidity of Exciton Polaritons Requires Strong Anisotropy, Phys. Rev. X 5, 011017 (2015).

[26] R. M. Stevenson, V. N. Astratov, M. S. Skolnick, D. M. Whittaker, M. Emam-Ismail, A. I. Tartakovskii, P. G. Savvidis, J. J. Baumberg, and J. S. Roberts, Continuous Wave Observation of Massive Polariton Redistribution by Stimulated Scattering in Semiconductor Microcavities, Phys. Rev. Lett. 85, 3680 (2000).

[27] J. J. Baumberg, P. G. Savvidis, R. M. Stevenson, A. I. Tartakovskii, M. S. Skolnick, D. M. Whittaker, and 
J. S. Roberts, Parametric Oscillation in a Vertical Microcavity: A Polariton Condensate or Micro-Optical Parametric Oscillation, Phys. Rev. B 62, R16247 (2000).

[28] A. Chiocchetta and I. Carusotto, Non-Equilibrium QuasiCondensates in Reduced Dimensions, Europhys. Lett. 102, 67007 (2013).

[29] K. Vogel and H. Risken, Quasiprobability Distributions in Dispersive Optical Bistability, Phys. Rev. A 39, 4675 (1989).

[30] M. H. Szymańska, J. Keeling, and P. B. Littlewood, MeanField Theory and Fluctuation Spectrum of a Pumped Decaying Bose-Fermi System across the Quantum Condensation Transition, Phys. Rev. B 75, 195331 (2007).

[31] D. F. Walls and G. J. Milburn, Quantum Optics (Springer, New York, 2007).

[32] D. M. Whittaker, Effects of Polariton-Energy Renormalization in the Microcavity Optical Parametric Oscillator, Phys. Rev. B 71, 115301 (2005).

[33] M. Wouters and I. Carusotto, Parametric Oscillation Threshold of Semiconductor Microcavities in the Strong Coupling Regime, Phys. Rev. B 75, 075332 (2007).

[34] F. M. Marchetti and M. H. Szymańska, Exciton Polaritons in Microcavities New Frontiers, Springer Series in SolidState Sciences, edited by V. Timofeev and D. Sanvitto (Springer-Verlag, Berlin, 2012), pp. 173-213.

[35] See Supplemental Material at http://link.aps.org/ supplemental/10.1103/PhysRevX.5.041028 for details on the numerical procedures.

[36] M. Wouters and I. Carusotto, Goldstone Mode of Optical Parametric Oscillators in Planar Semiconductor Microcavities in the Strong-Coupling Regime, Phys. Rev. A 76, 043807 (2007).

[37] C. Gardiner and P. Zoller, Quantum Noise: A Handbook of Markovian and Non-Markovian Quantum Stochastic Methods with Applications to Quantum Optics (Springer, New York, 2004), Vol. 56.

[38] I. Carusotto and C. Ciuti, Spontaneous MicrocavityPolariton Coherence across the Parametric Threshold:
Quantum Monte Carlo Studies, Phys. Rev. B 72, 125335 (2005).

[39] L. Giorgetti, I. Carusotto, and Y. Castin, Semiclassical Field Method for the Equilibrium Bose Gas and Application to Thermal Vortices in Two Dimensions, Phys. Rev. A 76, 013613 (2007).

[40] C. J. Foster, P. B. Blakie, and M. J. Davis, Vortex Pairing in Two-Dimensional Bose Gases, Phys. Rev. A 81, 023623 (2010).

[41] P. Drummond and D. Walls, Quantum Theory of Optical Bistability. I. Nonlinear Polarisability model, J. Phys. A 13, 725 (1980).

[42] L. M. Sieberer, S. D. Huber, E. Altman, and S. Diehl, Nonequilibrium Functional Renormalization for DrivenDissipative Bose-Einstein Condensation, Phys. Rev. B 89, 134310 (2014).

[43] R. Spano, J. Cuadra, C. Lingg, D. Sanvitto, M. D. Martin, P. R. Eastham, M. van der Poel, J. M. Hvam, and L. Viña, Build Up of Off-Diagonal Long-Range Order in Microcavity Exciton-Polaritons across the Parametric Threshold, Opt. Express 21, 10792 (2013).

[44] P. A. Murthy, I. Boettcher, L. Bayha, M. Holzmann, D. Kedar, M. Neidig, M. G. Ries, A. N. Wenz, G. Zürn, and S. Jochim, Observation of the Berezinskii-Kosterlitz-Thouless Phase Transition in an Ultracold Fermi Gas, Phys. Rev. Lett. 115, 010401 (2015).

[45] J. Keeling, F. Marchetti, M. Szymańska, and P. Littlewood, Collective Coherence in Planar Semiconductor Microcavities, Semicond. Sci. Technol. 22, R1 (2007).

[46] G. R. Dennis, J. J. Hope, and M. T. Johnsson, XMDS2: Fast, Scalable Simulation of Coupled Stochastic Partial Differential Equations, Comput. Phys. Commun. 184, 201 (2013).

[47] L. Ferrier, E. Wertz, R. Johne, D. D. Solnyshkov, P. Senellart, I. Sagnes, A. Lemaître, G. Malpuech, and J. Bloch, Interactions in Confined Polariton Condensates, Phys. Rev. Lett. 106, 126401 (2011). 